\title{
Systolic stretching of the ascending aorta
}

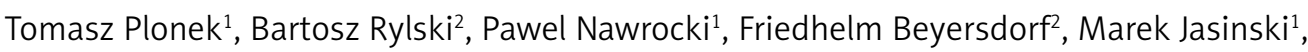 \\ Wiktor Kuliczkowski
}

${ }^{1}$ Department of Cardiac Surgery, Wroclaw Medical University, Wroclaw, Poland 2Department of Cardio-vascular Surgery, Heart Centre Freiburg University, Faculty of Medicine, University of Freiburg, Freiburg, Germany

${ }^{3}$ Department of Cardiology, Wroclaw Medical University, Wroclaw, Poland

Submitted: 22 August 2017

Accepted: 8 November 2017

Arch Med Sci 2021; 17 (1): 25-30

DOI: https://doi.org/10.5114/aoms.2019.82997

Copyright $\odot 2019$ Termedia \& Banach

\author{
Corresponding author: \\ Tomasz Plonek MD, PhD \\ Department of \\ Cardiac Surgery \\ Wroclaw Medical \\ University \\ 213 Borowska St \\ 50-556 Wroclaw, Poland \\ Phone: +48 602127941 \\ E-mail: tomaszplonek@gmail. \\ com
}

\begin{abstract}
Introduction: Longitudinal stretching of the aorta due to systolic heart motion contributes to the stress in the wall of the ascending aorta. The objective of this study was to assess longitudinal systolic stretching of the aorta and its correlation with the diameters of the ascending aorta and the aortic root. Material and methods: Aortographies of 122 patients were analyzed. The longitudinal systolic stretching of the aorta caused by the contraction of the heart during systole and the maximum dimensions of the aortic root and ascending aorta were measured in all patients.

Results: The maximum dimension of the aortic root was on average 34.9 $\pm 4.5 \mathrm{~mm}$ and the mean diameter of the ascending aorta was $33.9 \pm 5.4$ $\mathrm{mm}$. The systolic aortic stretching negatively correlated with age $(r=-0.49$, $p<0.001)$ and the diameter of the tubular ascending aorta $(r=-0.44$, $p<0.001)$. There was no significant correlation between the stretching and the dimension of the aortic root $(r=-0.11, p=0.239)$. There was a statistically significant $(p<0.001)$ difference in the longitudinal aortic stretching values between patients with a normal aortic valve $(10.6 \pm 3.1 \mathrm{~mm})$ and an aortic valve pathology $(8.0 \pm 3.2 \mathrm{~mm}$ in all patients with an aortic valve pathology; $7.5 \pm 4.3 \mathrm{~mm}$ in isolated aortic stenosis, $8.5 \pm 2.9 \mathrm{~mm}$ in the case of isolated insufficiency, $8.2 \pm 2.8 \mathrm{~mm}$ for valves that were both stenotic and insufficient). Conclusions: Systolic aortic stretching negatively correlates with the diameter of the tubular ascending aorta and the age of the patients, and does not correlate with the diameter of the aortic root. It is lower in patients with an aortic valve pathology.
\end{abstract}

Key words: aorta, dissection, aneurysm.

\section{Introduction}

The maximum diameter and the rapid growth of the aortic dimensions are the main factors considered when qualifying patients for surgery [1-3]. Other risk factors such as a family history of aortic dissection, genetic disorders and severe uncontrollable hypertension also are used to assess the risk of aortic complications [2]. It is believed that the risk of aortic dissection rapidly increases when the diameter of the vessel exceeds 50-60 mm [4]. On the other hand, a recently published multicenter study proved that most aortas had a diameter roughly $40 \mathrm{~mm}$ shortly before dissection [5]. The dissection led to a significant increase in the vessel diameter up to $50-60 \mathrm{~mm}$. Hence, patients with moderately 
dilated aortas are also at risk of aortic dissection. People who are genetically predisposed to aortic dissection can be offered preventive surgery at an early stage $[2,6]$. However, considering the risk of aortic operations, not all patients with moderately dilated aortas should undergo this type of surgery [7]. A new diagnostic tool is necessary to select patients who are at risk of dissection and thus require more aggressive treatment among those whose aortas are moderately dilated.

Aortic wall stiffness is a factor that is believed to contribute to a higher risk of dissection $[8,9]$. The circumferential stress that is predominantly caused by blood pressure can be assessed through measuring the aortic distensibility and pulse wave velocity. However, to date, no diagnostic examinations have been used to measure the longitudinal stiffness of the wall of the ascending aorta.

There are three main dynamic mechanical factors that contribute to stress in the ascending aorta: arterial blood pressure, the characteristics of the blood flow and systolic up-and-down movement of the aorta (systolic aortic stretching - SAS) during the heart cycle [10]. The latter is believed to significantly impact longitudinal stress of the ascending aorta [11]. However, there are no clinical studies that assess the correlation of this parameter with the aortic diameter, aortic wall stiffness or incidence of acute aortic syndromes.

The aim of this study was to define the normal values of the up-and-down movement of the aorta during the heart cycle (SAS) and to analyze how the aortic diameter, patient age, height and aortic valve pathology correlate with this parameter.

\section{Material and methods}

\section{Patients}

The aortographies of 310 patients were selected for primary assessment and those that were difficult to assess objectively (arrhythmia, not enough contrast, aortic motion due to the move- ment of the diaphragm, bad visualization of the aorta) were excluded. One hundred and twenty two patients, mainly with aortic valve stenosis $(n=43,35 \%)$ and/or coronary artery disease ( $n=38,31 \%$ ) were eligible for the final analysis. The gender, age, body surface area (BSA) and clinical data (aortic valve pathology, left ventricular ejection fraction) were collected by retrospective review of patient records.

\section{Methods}

The aortographies were measured at a projection resembling the one used in the transcatheter aortic valve implantation - all three sinuses were visible at the same level $($ LAO $\approx$ 10 , caudal $\approx-10$ ). The examinations were performed by injecting $40 \mathrm{ml}$ of the lomeron 350 contrast agent (Bracco Imaging S.p.A., Bioindustry Park, Via Ribes 5, 10010 Colleretto Giacosa, Italy) directly into the aortic root. The radiation dose for the aortography ranged from 5 to 10 mGy. The diameters were measured at the level of the aortic root, sinotubular junction (STJ) and tubular ascending aorta. A systolic-diastolic longitudinal translocation of the aorta (systolic aortic stretching - SAS) caused by the contraction of the heart during systole was measured at the level of the ventriculo-aortic junction (Figure 1). This was correlated with the maximum dimension of the aortic root and ascending aorta, the patient age, the body surface area (BSA) and the left ventricular ejection fraction (EF). Moreover, the systolic aortic stretching was compared between patients with and without an aortic valve pathology.

\section{Statistical analysis}

The normality of distribution was assessed using the Shapiro-Wilk test. The Pearson correlation was used for variables with a normal distribution and the Spearman rank R correlation was used for
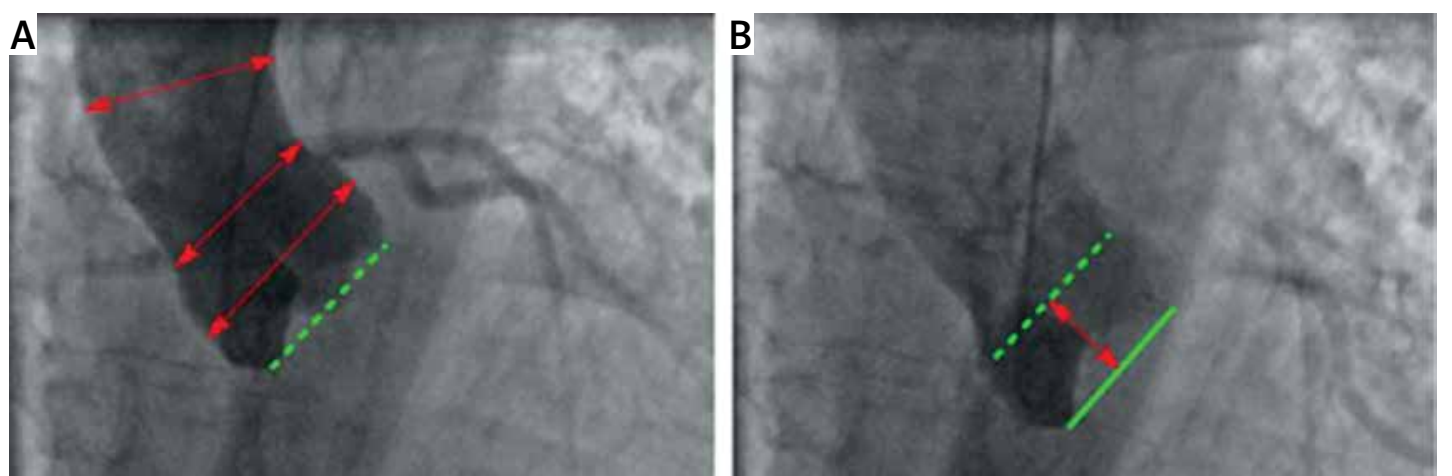

Figure 1. Angiograms of the aorta (aortography) during diastole (A) and systole (B). The measurements were taken at the level of the aortic root, sinotubular junction and ascending aorta (red arrows on the A). The systolic aortic stretching (SAS) is the distance in $\mathrm{mm}$ that the virtual basal ring is pulled by the heart from diastole (dotted green line on the A) to peak systole (solid green line on the B) 


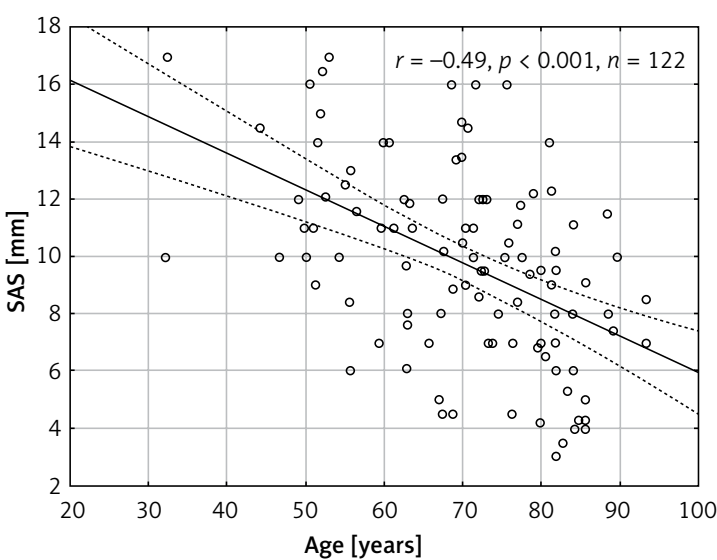

Figure 2. Scatterplot presenting the correlation between age of patients and systolic aortic stretching (SAS)

variables without a normal distribution. The differences in SAS between patients with and without an aortic valve pathology were assessed using Student's t-test. All analyses were performed using Dell Statistica 12 software (Dell, USA). The study was approved by the ethics committee of Wroclaw Medical University.

\section{Results}

The mean age of the patients was $70.5 \pm 13$ years and 54 (44\%) patients were male. The aorta was stretched longitudinally during systole (SAS) by $9.6 \pm 3.4 \mathrm{~mm}$. The SAS did not differ between males and females $(9.7 \pm 3.6 \mathrm{~mm}$ vs. $9.6 \pm 3.2 \mathrm{~mm}$, $p=0.89)$ and it negatively correlated with age $(r=-0.49, p<0.001)$ (Figure 2). There was a statistically significant correlation between SAS and patient height $(r=0.2, p=0.038$ ) (Figure 3). However, no significant correlations between SAS and weight $(r=0.0212, p=0.828)$, body mass index $(r=-0.08, p=0.407)$, and BSA $(r=0.099, p=$ $0.309)$ were observed. The patient characteristics are presented in Table I.

\section{Diameters of the aorta}

The maximum dimension of the aortic root was on average $34.9 \pm 4.5 \mathrm{~mm}$ and the mean maximum diameter of the ascending aorta was $33.9 \pm 5.4 \mathrm{~mm}$. There was a significant negative correlation between SAS and the diameter of the ascending aorta $(r=-0.44, p<0.001)$ (Figure 4) but no significant correlation between SAS and the dimension of the root $(r=-0.11, p=0.239)$ was observed (Figure 5 ). There was a statistically significant difference $(p=0.015)$ in SAS values between patients with an ascending aorta diameter exceeding $40 \mathrm{~mm}$ $(7.7 \pm 2.6 \mathrm{~mm})$ and those with an ascending aorta diameter of $40 \mathrm{~mm}$ or less $(9.9 \pm 3.4 \mathrm{~mm})$. No significant difference $(p=0.52)$ was observed between patients with an aortic root dimension

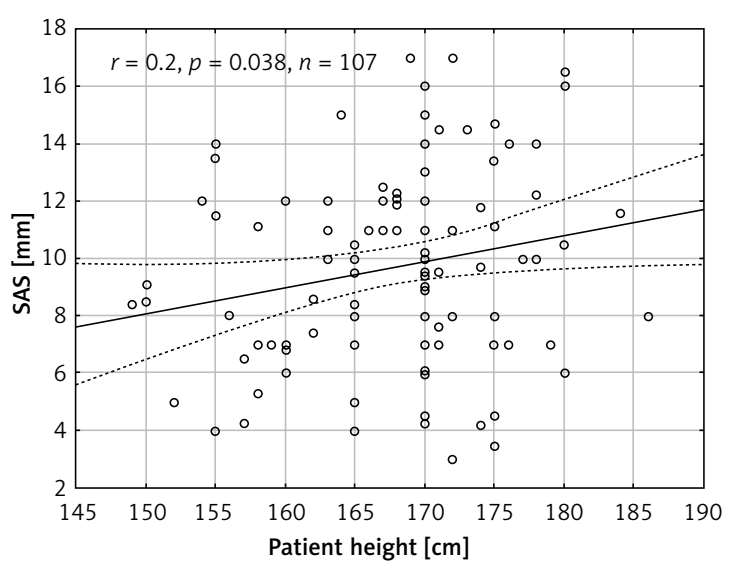

Figure 3. Scatterplot presenting the correlation between patient height and systolic aortic stretching (SAS)

Table I. Characteristics of patients

\begin{tabular}{|lc|}
\hline Parameter & Value \\
\hline Age [years] & $70.5 \pm 13$ \\
\hline Gender & $\begin{array}{c}54(44 \%) \\
\text { males }\end{array}$ \\
\hline Patient height $[\mathrm{cm}]$ & $168.1 \pm 7.5$ \\
\hline Patient weight $[\mathrm{kg}]$ & $72.3 \pm 13.2$ \\
\hline Body surface area [m²] & $1.85 \pm 0.16$ \\
\hline Body mass index [kg/m $\left.{ }^{2}\right]$ & $26.6 \pm 4.5$ \\
\hline Left ventricular ejection fraction (\%) & $53 \pm 7.7$ \\
\hline Dimension of aortic root [mm] & $34.9 \pm 4.5$ \\
\hline Diameter of ascending aorta [mm] & $33.9 \pm 5.4$ \\
\hline
\end{tabular}

exceeding $40 \mathrm{~mm}(9.04 \pm 2.89)$ and measuring $40 \mathrm{~mm}$ or less $(9.7 \pm 3.5 \mathrm{~mm})$.

\section{Left ventricular ejection fraction}

The mean left ventricular ejection fraction (LVEF) was $53 \pm 7.7 \%$. A statistically significant

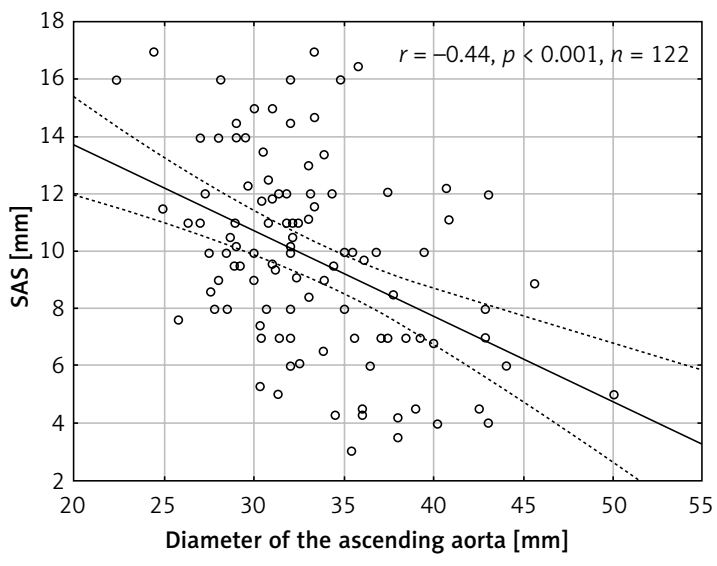

Figure 4. Scatterplot presenting the correlation between diameter of the ascending aorta and systolic aortic stretching (SAS) 


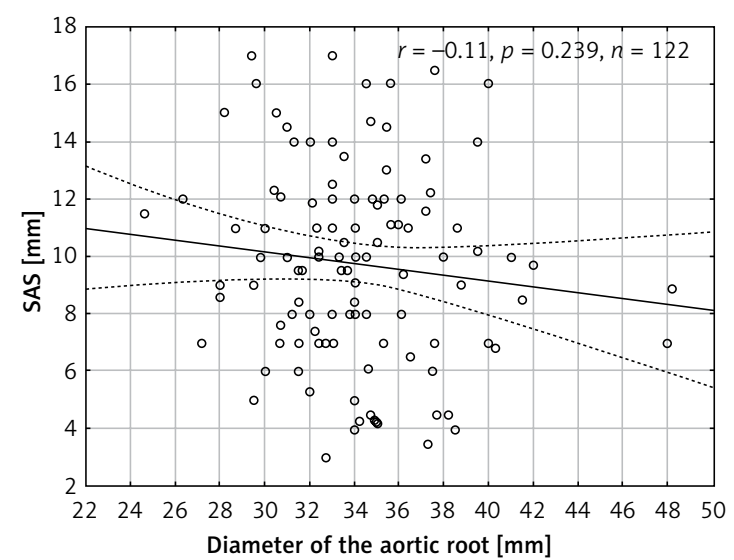

Figure 5. Scatterplot presenting the correlation between diameter of the aortic root and systolic aortic stretching (SAS)

correlation was observed between SAS and the left ventricular ejection fraction $(r=0.307, p=$ 0.001) (Figure 6). Moreover, there was a statistically significant difference $(p=0.002)$ between the patients with normal ejection fraction (LVEF $>50 \% ; 10.04 \pm 3.4 \mathrm{~mm}$ ) and those with impaired EF $(7.4 \pm 2.4 \mathrm{~mm})$.

\section{Aortic valve pathology}

Forty-three (35\%) patients had an aortic valve pathology (aortic insufficiency: 11 (9\%) patients; aortic stenosis: 14 (11\%) patients; aortic stenosis with aortic insufficiency: 18 (15\%) patients). There was a statistically significant $(p<0.001)$ difference in the SAS values between patients with a normal aortic valve $(10.6 \pm 3.1 \mathrm{~mm})$ and an aortic valve pathology $(8.0 \pm 3.2 \mathrm{~mm})$. Mean SAS values in patients with isolated aortic stenosis, isolated aortic insufficiency, and in patients whose aortic valves were both stenotic and insufficient were $7.5 \pm 4.3 \mathrm{~mm}$, $8.5 \pm 2.9 \mathrm{~mm}$ and $8.2 \pm 2.8 \mathrm{~mm}$, respectively.

\section{Discussion}

This is the first study that assesses the correlation between the systolic stretching of the ascending aorta (SAS) and the diameters of the aorta, patient age, height, weight and left ventricular ejection fraction. The findings of this study can be summarized as follows: (i) systolic aortic stretching negatively correlates with the diameter of the tubular ascending aorta and patient age, (ii) SAS correlates with patient height and the left ventricular ejection fraction, (iii) SAS does not correlate with the maximum dimension of the aortic root and patient weight, (iv) SAS is lower in patients with an aortic valve pathology.

\section{Relationship to previous studies}

The aorta loses its elasticity and increases in diameter with age [12-14]. In our study, SAS neg-

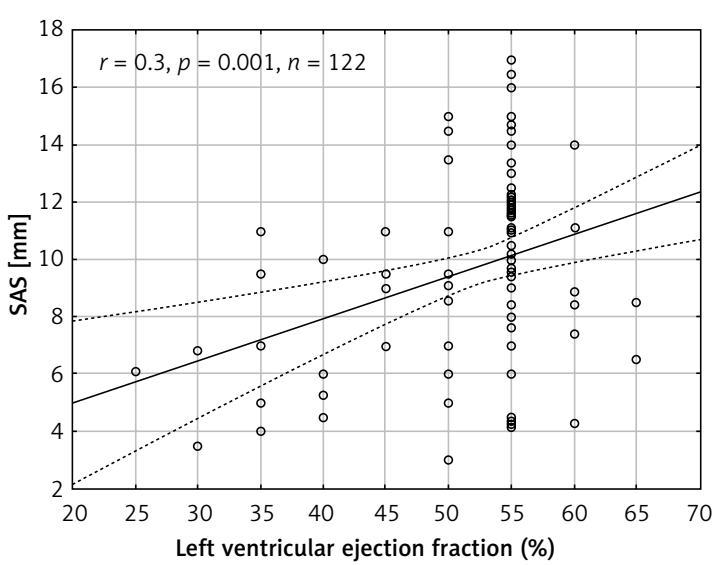

Figure 6. Scatterplot presenting the correlation between left ventricular ejection fraction and systolic aortic stretching (SAS)

atively correlated with age and the diameter of the ascending aorta. One of the explanations for this phenomenon is that the contracting heart is not able to pull and stretch the stiffer aorta to the same distance as in the case of a normal elastic aorta. Surprisingly, no correlation was found between SAS and the aortic root dimension. This may be explained by the fact that the aortic root is a complex structure with aortic valve leaflets and coronary ostia and its biomechanical properties significantly differ from those of the tubular ascending aorta [15].

Patients with an aortic valve pathology had lower SAS values than patients with a normal aortic valve function. This phenomenon may be caused by the fact that aortic stenosis was the predominant aortic valve pathology. Aortic stenosis may have caused concentric left ventricular hypertrophy, which may have reduced the distance the heart pulled the aorta during systole.

A weak correlation $(r=0.2, p=0.038)$ was found between SAS and patient height. This finding may be explained by the fact that taller people usually have bigger hearts and longer ascending aortas. No significant correlation was observed between SAS and patient weight, body surface area or body mass index.

\section{Aortic wall elasticity}

There are a few parameters that are taken into account when qualifying patients with dilated aortas for surgery. The most important of these is the diameter of the aorta, its relation to the patient height and body surface area, as well as the increase in the diameter over a specific period of time [2]. However, recent studies suggest that the aortic diameter alone is not a good predictor of the risk of an aortic dissection. New parameters such as blood flow patterns, the geometry of the proximal segments of the aorta, i.e. the angles between its segments, or aortic elongation are stud- 
ied to define new risk factors for aortic dissection [16-18]. One of these parameters - impaired aortic wall elasticity and subsequent increase in aortic wall stress - may be crucial to aid in the diagnosis of patients with potentially "high risk aortas".

The intimal layer can be damaged and lead to dissection when the stress in the aortic wall exceeds its tensile strength. The stress can be elevated due to the increase in aortic wall stiffness or blood pressure. According to LaPlace's law, the circumferential tension in the vessel wall is directly proportional to its radius. Therefore, the tension and stress in the wall of an aneurysm are higher than in the non-dilated vessel under the same pressure. It is hypothesized that the intima is more likely to rupture when the vessel loses its elasticity and cannot adapt to the pulsatile blood pressure.

One of the methods to assess arterial stiffness is the measurement of pulse wave velocity using applanation tonometry [19] and echocardiography [20]. The regional circumferential stiffness in the ascending aorta can be measured using magnetic resonance imaging [21]. Potentially, such measurements could also be performed on aortas using the speckle-tracking analysis in echocardiography, similarly to the measurements of left ventricular strain [22, 23]. Aortic stiffness is higher in patients predisposed to aortic aneurysms and dissection compared to healthy subjects [24, 25]. Moreover, according to the results of a meta-analysis, aortic stiffness measured by pulse wave velocity (PWV) is an independent predictor of adverse cardiovascular events and all-cause mortality [26]. In addition, it can be a predictor of the risk of aortic dilatation and dissection in patients with Marfan syndrome [8]

All the above-mentioned diagnostic methods assess circumferential stiffness/elasticity. The aortic wall is also subjected to longitudinal stress, caused by the longitudinal up-and-down movement of the heart $[11,27]$. A biomechanical study by Beller et al. suggested that the longitudinal aortic root motion had a bigger impact on wall stress in the ascending aorta and aortic root than blood pressure [27]. To date, there is no commonly used diagnostic parameter that corresponds to the longitudinal aortic stiffness. Such a parameter could help in the assessment of the risk of aortic dissection. In most patients, the intimal tears are circumferential and are most likely caused by longitudinal stretch of the aorta $[27,28]$.

In our opinion, SAS may be an important parameter for the assessment of longitudinal aortic wall stiffness. This statement is supported by the biomechanical studies which suggest that a lack of aortic wall elasticity and high circumferential stress caused by longitudinal stretch of the aorta may play a role in type A aortic dissection [11, 27].
The SAS was measured based on aortographies. Aortography is an invasive method and is not routinely performed in all patients. A study that analyzes the SAS with noninvasive diagnostic methods, i.e. magnetic resonance imaging or echocardiography, is necessary to translate the study into clinical practice. Moreover, a larger population-based study is necessary to determine normal values for specific age groups, gender, height/body surface area and aortic diameter.

In conclusion, systolic aortic stretching negatively correlates with the diameter of the tubular ascending aorta and age and does not correlate with the diameter of the aortic root. The SAS is lower in patients with aortic valve pathologies. Further studies are needed to assess systolic aortic stretching in aortic aneurysms, its correlation with aortic wall stiffness and the incidence of aortic dissection.

\section{Acknowledgments}

The study was financed by a Wroclaw Medical University statutory grant (no. ST-829) .

The manuscript was presented during the $65^{\text {th }}$ International Congress of the European Society for Cardiovascular and Endovascular Surgery (Belgrade, Serbia, 21-23 April 2016)

\section{Conflict of interest}

The authors declare no conflict of interest.

\section{References}

1. Elefteriades JA, Farkas EA. Thoracic aortic aneurysm clinically pertinent controversies and uncertainties. J Am Coll Cardiol 2010; 55: 841-57.

2. Erbel R, Aboyans V, Boileau C, et al. 2014 ESC Guidelines on the diagnosis and treatment of aortic diseases: document covering acute and chronic aortic diseases of the thoracic and abdominal aorta of the adult. The Task Force for the Diagnosis and Treatment of Aortic Diseases of the European Society of Cardiology (ESC). Eur Heart J 2014; 35: 2873-926.

3. Hiratzka LF, Bakris GL, Beckman JA, et al. 2010 ACCF/ AHA/AATS/ACR/ASA/SCA/SCAI/SIR/STS/SVM guidelines for the diagnosis and management of patients with Thoracic Aortic Disease: a report of the American College of Cardiology Foundation/American Heart Association Task Force on Practice Guidelines, American Association for Thoracic Surgery, American College of Radiology, American Stroke Association, Society of Cardiovascular Anesthesiologists, Society for Cardiovascular Angiography and Interventions, Society of Interventional Radiology, Society of Thoracic Surgeons, and Society for Vascular Medicine. Circulation 2010; 121 : e266-369.

4. Elefteriades JA. Beating a sudden killer. Sci Am 2005; 293: 64-71.

5. Rylski B, Blanke P, Beyersdorf F, et al. How does the ascending aorta geometry change when it dissects? J Am Coll Cardiol 2014; 63: 1311-9. 
6. Treasure T, Takkenberg JJ, Golesworthy T, et al. Personalised external aortic root support (PEARS) in Marfan syndrome: analysis of 1-9 year outcomes by intention-to-treat in a cohort of the first 30 consecutive patients to receive a novel tissue and valve-conserving procedure, compared with the published results of aortic root replacement. Heart 2014; 100: 969-75.

7. Williams JB, Peterson ED, Zhao Y, et al. Contemporary results for proximal aortic replacement in North America. J Am Coll Cardiol 2012; 60: 1156-62.

8. Vitarelli A, Conde $\mathrm{Y}$, Cimino E, et al. Aortic wall mechanics in the Marfan syndrome assessed by transesophageal tissue Doppler echocardiography. Am J Cardiol 2006; 97: 571-7

9. Rajzer M, Wojciechowska W, Kameczura T, et al. The effect of antihypertensive treatment on arterial stiffness and serum concentration of selected matrix metalloproteinases. Arch Med Sci 2017; 13: 760-70.

10. Martufi G, Forneris A, Appoo JJ, Di Martino ES. Is there a role for biomechanical engineering in helping to elucidate the risk profile of the thoracic aorta? Ann Thorac Surg 2016; 101: 390-8.

11. Plonek T, Rylski B, Dumanski A, Siedlaczek P, Kustrzycki W. Biomechanical analysis of wrapping of the moderately dilated ascending aorta. J Cardiothorac Surg 2015; $10: 106$

12. Ohyama Y, Teixido-Tura G, Ambale-Venkatesh B, et al. Ten-year longitudinal change in aortic stiffness assessed by cardiac MRI in the second half of the human lifespan: the multi-ethnic study of atherosclerosis. Eur Heart J Cardiovasc Imaging 2016; 17: 1044-53.

13. Hickson SS, Butlin M, Graves M, et al. The relationship of age with regional aortic stiffness and diameter. JACC Cardiovasc Imaging 2010; 3: 1247-55.

14. Ferrara A, Morganti S, Totaro P, Mazzola A, Auricchio F. Human dilated ascending aorta: mechanical characterization via uniaxial tensile tests. J Mech Behav Biomed Mater 2016; 53: 257-71.

15. Contino $M$, Mangini A, Lemma MG, et al. A geometric approach to aortic root surgical anatomy. Eur J Cardiothorac Surg 2016; 49: 93-100.

16. Girdauskas E, Rouman M, Disha K, et al. Aortopathy in patients with bicuspid aortic valve stenosis: role of aortic root functional parameters. Eur J Cardiothorac Surg 2016; 49: 635-43; discussion 43-4.

17. Kruger T, Forkavets O, Veseli K, et al. Ascending aortic elongation and the risk of dissection. Eur J Cardiothorac Surg 2016; 50: 241-7.

18. Numata S, Itatani K, Kanda K, et al. Blood flow analysis of the aortic arch using computational fluid dynamics. Eur J Cardiothorac Surg 2016; 49: 1578-85.

19. Nelson MR, Stepanek J, Cevette M, Covalciuc M, Hurst RT, Tajik AJ. Noninvasive measurement of central vascular pressures with arterial tonometry: clinical revival of the pulse pressure waveform? Mayo Clin Proc 2010; 85: 460-72.

20. Jiang B, Liu B, McNeill KL, Chowienczyk PJ. Measurement of pulse wave velocity using pulse wave Doppler ultrasound: comparison with arterial tonometry. Ultrasound Med Biol 2008; 34: 509-12.

21. Redheuil A, Yu WC, Wu CO, et al. Reduced ascending aortic strain and distensibility: earliest manifestations of vascular aging in humans. Hypertension 2010; 55: 319-26.

22. Karatolios K, Wittek A, Nwe TH, et al. Method for aortic wall strain measurement with three-dimensional ultra- sound speckle tracking and fitted finite element analysis. Ann Thorac Surg 2013; 96: 1664-71.

23. Wierzbowska-Drabik K, Plewka M, Kasprzak JD. Variability of longitudinal strain in left ventricular segments supplied by non-stenosed coronary artery: insights from speckle tracking analysis of dobutamine stress echocardiograms in patients with high coronary risk profile. Arch Med Sci 2017; 13: 82-92.

24. Nistri S, Grande-Allen J, Noale M, et al. Aortic elasticity and size in bicuspid aortic valve syndrome. Eur Heart 2008; 29: 472-9.

25. Harada K, Yasuoka K, Shimada Y. Usefulness of tissue doppler imaging for assessing aortic wall stiffness in children with the Marfan syndrome. Am J Cardiol 2004; 93: 1072-5.

26. Vlachopoulos C, Aznaouridis K, Stefanadis C. Prediction of cardiovascular events and all-cause mortality with arterial stiffness: a systematic review and meta-analysis. J Am Coll Cardiol 2010; 55: 1318-27.

27. Beller CJ, Labrosse MR, Thubrikar MJ, Robicsek F. Role of aortic root motion in the pathogenesis of aortic dissection. Circulation 2004; 109: 763-9.

28. Hirst AE Jr, Johns VJ Jr, Kime SW Jr. Dissecting aneurysm of the aorta: a review of 505 cases. Medicine (Baltimore) 1958; 37: 217-79. 